\title{
Tearing mode physics studies applying the dynamic ergodic divertor on TEXTOR
}

\author{
H R Koslowski ${ }^{1}$, E Westerhof ${ }^{2}$, M de Bock $^{2}$, I Classen $^{2}$, R Jaspers ${ }^{2}$, \\ Y Kikuchi ${ }^{1}$, A Krämer-Flecken ${ }^{1}$, A Lazaros ${ }^{2}$, Y Liang ${ }^{1}$, K Löwenbrück ${ }^{1}$,

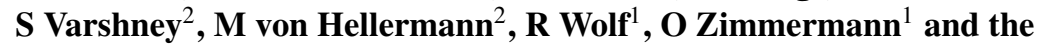 \\ TEXTOR team \\ ${ }^{1}$ Forschungszentrum Jülich GmbH, Institut für Plasmaphysik, Association EURATOM-FZ \\ Jülich, D-52425 Jülich, Germany ${ }^{3}$ \\ 2 FOM-Institute for Plasmaphysics 'Rijnhuizen', Association EURATOM-FOM, PO Box 1207, \\ 3430 BE Nieuwegein, The Netherlands ${ }^{3}$ \\ E-mail: h.r.koslowski@fz-juelich.de
}

Received 23 June 2006

Published 8 November 2006

Online at stacks.iop.org/PPCF/48/B53

\begin{abstract}
The dynamic ergodic divertor (DED) on the TEXTOR tokamak allows for the reproducible destabilization of the $m / n=2 / 1$ tearing mode which is phase locked to the external static or rotating perturbation field. In combination with its flexible heating systems (co- and counter-neutral beam injection, ion cyclotron resonance heating, electron cyclotron resonance heating $(\mathrm{ECRH})$ with steerable launcher) dedicated experiments to study the mode onset, properties of large islands and mode stabilization can be performed. The dependence of the mode excitation threshold (field penetration) on the plasma rotation shows a resonance character, with minimum threshold when the external perturbation frequency matches the MHD frequency of the 2/1 mode. Mode stabilization by ECRH heating shows that for the TEXTOR plasma heating is more effective than the current drive in O-point. Extrapolation to ITER yields a significant contribution to the mode suppression originating from the temperature increase within the island. Alfvén-like modes, which have been previously identified in the vicinity of large islands on FTU (Buratti et al 2005 Nuclear Fusion 45 1446), are found to be created already before island formation above a certain threshold of the externally applied perturbation field.
\end{abstract}

\footnotetext{
${ }^{3}$ Members in the Trilateral Euregio Cluster (TEC). 


\section{Introduction}

Tearing modes in tokamak plasmas limit the achievable confinement and can initiate disruptions. The sources of the excitation of tearing modes are (i) an unfavourably shaped current profile [1], (ii) non-axisymmetric error fields [2] arising from imperfections in the construction or alignment of the magnetic field coils and (iii) pressure driven contributions causing so-called neo-classical tearing modes (NTMs) [3]. Whereas NTMs mainly limit the fusion performance of a tokamak because the threshold for their destabilization occurs below the ideal beta limit, the error field mode generation occurs predominantly at low plasma density and beta during the starting phase of the plasma. Of special importance is the $m / n=2 / 1$ tearing mode which normally has a small negative $\Delta^{\prime}$ and can easily be destabilized. It grows to a quite large island size and causes a considerable loss of confinement, or even triggers a disruption when the mode locks to the wall.

Experiments on various tokamaks [4-8] showed that a relative amplitude of the radial error field normalized to the toroidal magnetic field of the order of $B_{r} / B_{\mathrm{t}}=5 \times 10^{-5}$ may be sufficient to excite the $2 / 1$ mode in ITER. Since this is of the same order as the anticipated intrinsic error field, an error field correction system capable of correcting the intrinsic errors has to be provided.

Most of the experiments on error field thresholds were done in Ohmic or L-mode plasmas. DIII-D experiments at higher beta revealed an increasing susceptibility for error fields to generate tearing modes [5].

NTMs are triggered by sufficiently large magnetic perturbations, mainly from core MHD events like sawteeth. Once destabilized the loss of bootstrap current in the island (O-point) drives the mode to its saturated size. Suppression of NTMs requires replacement of the lost current by driving a non-inductive current. The preferred method for this is electron cyclotron current drive (ECCD) due to its high spatial localization.

The TEXTOR tokamak is equipped with the dynamic ergodic divertor (DED) [9] which consists of 16 coils inside the vacuum vessel wound helically around the high-field side of the torus, having the same pitch as the field lines of the $q=3$ surface. The coil system can be wired to operate in base modes $m / n=12 / 4,6 / 2$ and 3/1. The DED can be operated with a static (dc) field or with a rotating (ac) field with frequencies of several $\mathrm{kHz}$ and the toroidal direction of rotation either in (co) or opposite (counter) to the plasma current. The external perturbation field of the DED allows for reproducible excitation of $m / n=2 / 1$ tearing modes [10] when operated in the $3 / 1$ configuration due to a strong $m / n=2 / 1$ sideband perturbation. The tearing mode is a generated frequency locked to the external perturbation field, i.e. it is locked in the tokamak frame for static (dc) operation of the DED and it rotates toroidally with the same frequency when dynamic (ac) operation is applied. This behaviour is extremely reproducible and allows for detailed studies of error field penetration in dependence on various plasma parameters.

TEXTOR is equipped with two neutral beam injectors, one in the co-current direction and the other in the counter-current direction. The power from each neutral beam can be precisely controlled by movable apertures in the beam ducts [11]. In combination with the ion cyclotron resonance heating system the heating power (beta) and the momentum input to the plasma (rotation) can be selected independently. In addition, TEXTOR has a $140 \mathrm{GHz}$ gyrotron with a steerable launcher [12]. Electron cyclotron resonance heating (ECRH) or ECCD can be applied to investigate suppression of 2/1 islands generated with the DED. All together allow for welldefined experiments in order to study the physics of tearing mode excitation by perturbation fields and suppression by heating or current drive. 


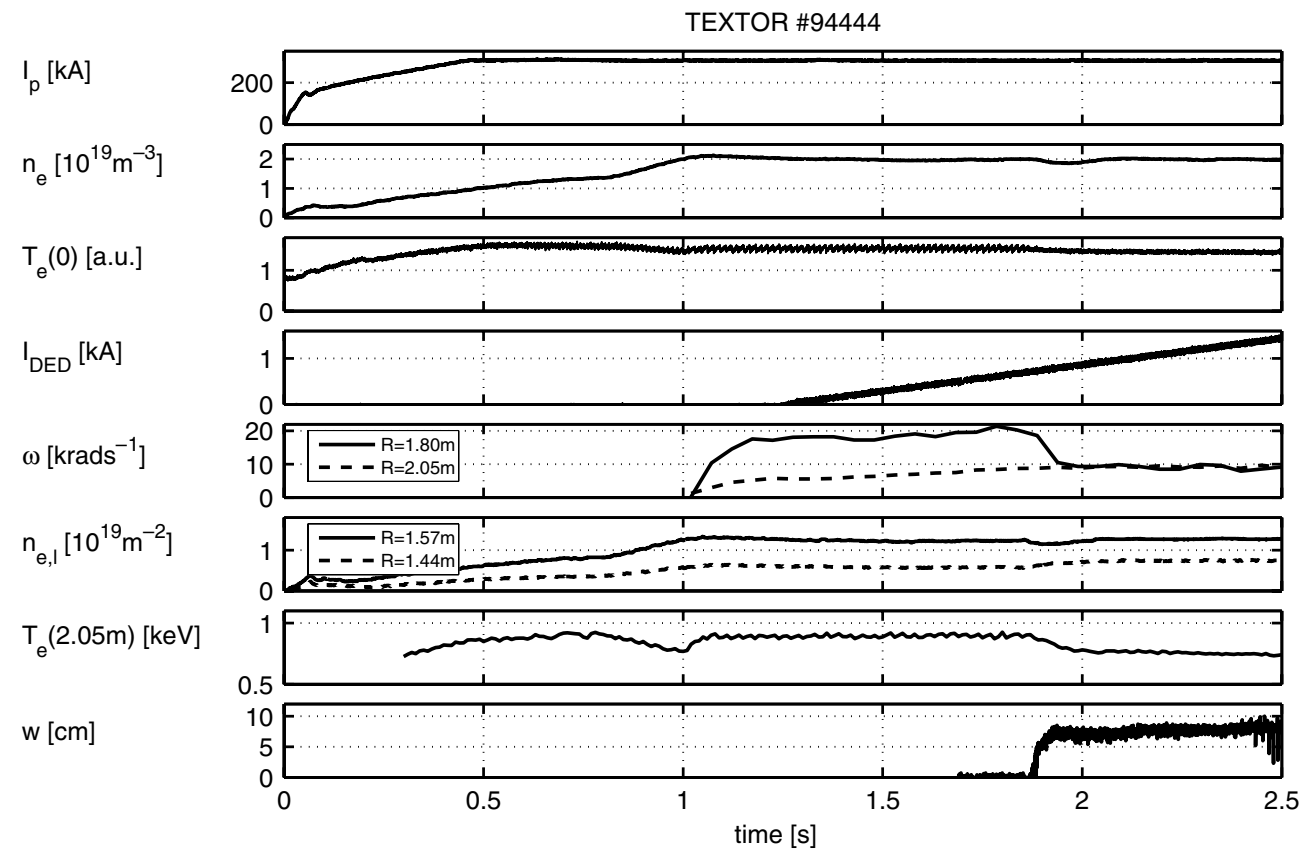

Figure 1. Typical example of a discharge which has been designed for field penetration studies. (For a detailed explanation of the shown traces see text.)

\section{Mode excitation with the DED}

A typical example of a discharge to study the parametric dependence of error field mode generation is shown in figure 1. The traces shown in the figure from top to bottom are (i) the plasma current, (ii) the line-averaged electron density, (iii) the central electron temperature, (iv) the current in the DED coils, (v) the angular frequency of the plasma rotation in the centre and close to the $q=2$ surface, (vi) the line-integrated electron density measured along two chords inside and outside of $r_{q=2}$, (vii) the local electron temperature close to $q=2$ and (viii) the island width determined by an SXR camera. At $t=1.75 \mathrm{~s}$ an $m / n=2 / 1$ tearing mode is created in the plasma when the DED current exceeds the threshold value (about $0.8 \mathrm{kA}$ for this specific plasma). The mode is generated locked in the tokamak frame and does not rotate. The SXR camera yields a saturated island width of about $8 \mathrm{~cm}$. The creation of the island can be seen in the line integrated electron densities on the high-field side of the torus which increase at $R=1.44 \mathrm{~m}$ and decrease at $R=1.57 \mathrm{~m}$, indicating a flattening of the electron density profile due to the enhanced transport around the island. The electron temperature at $R=2.05 \mathrm{~m}$ (measured at the low-field side) decays, too. Most striking is the change in the angular frequencies which exhibit, prior to the penetration of the error field and the formation of the locked mode, a braking of the central plasma rotation [13]. The same values of the angular frequency at different radii indicate that the central part of the plasma rotates like a rigid body. A slight decrease is seen in the central electron temperature, whereas the decrease in the line-averaged electron density has only a short duration due to the action of the feedback control which restores the pre-programmed density. The edge safety factor, $q_{\mathrm{a}}$, has been set to 4.5 in cylindrical approximation because for this and larger values the locked mode does not lead to a disruption. 

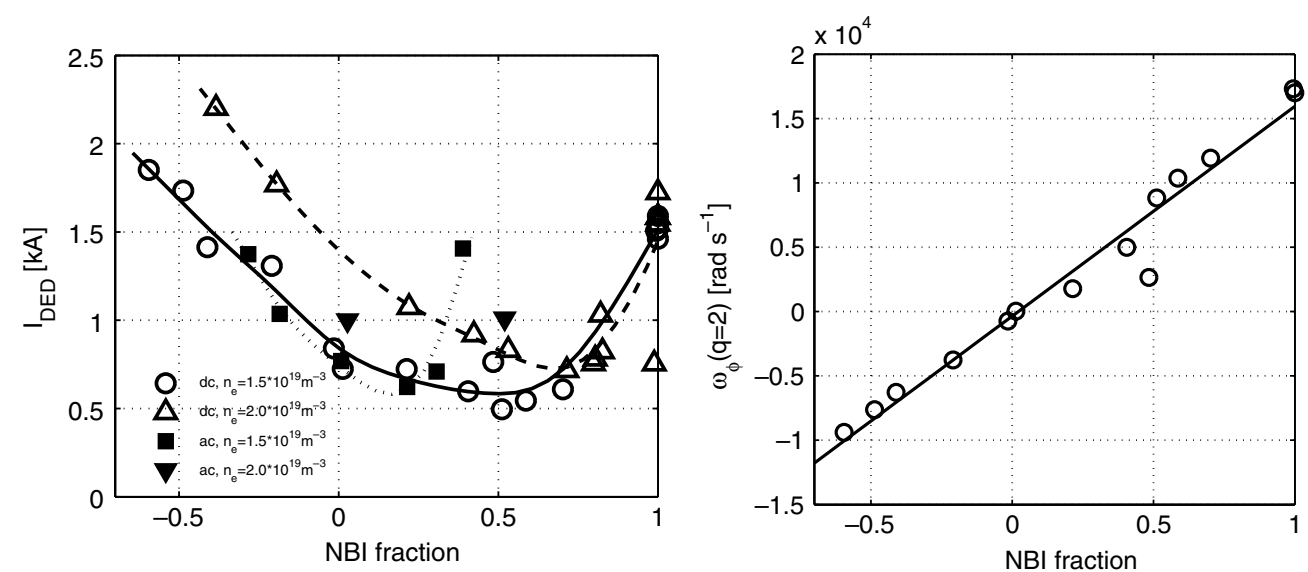

Figure 2. (left) Mode onset thresholds (shown is the equivalent current in the DED coils) versus the net momentum input to the plasma (expressed as the NBI fraction. (right) Angular velocity at $q=2$ versus the NBI fraction.

The toroidal and poloidal mode numbers have been determined to be $n=1$ and $m=2$ by phase comparison between adjacent magnetic pick-up coils from toroidal and poloidal arrays after the DED was switched off and the mode started rotating.

The onset threshold for the locked mode shows a linear dependence on the electron density and the critical field normalized to the toroidal field depends inversely on the toroidal field, $B_{r} / B_{\Phi} \propto 1 / B_{\Phi}[10]$. This behaviour agrees well with previous error field mode onset studies performed on other tokamaks.

The dependence of the mode onset threshold on beta, determined in a series of experiments with an increasing amount of ICRH heating power, shows a strong increase. This behaviour is different from earlier experiments on the JET tokamak which show only a small influence of ICRH heating on the mode threshold [6].

\section{Dependence of the error field penetration on plasma rotation}

A series of discharges similar to the one shown in figure 1 has been performed where before the application of the DED both NBI systems were switched on at a constant level of total power, but with varying relative fractions. The DED has been operated in dc and with a $1 \mathrm{kHz}$ ac field where the direction of rotation was chosen to be in opposite direction to the plasma current. For both operating modes of the DED the experiment was done at two different electron densities. The results from these different scans are summarized in the left part of figure 2. The critical DED coil current (where the ac case has been scaled to an equivalent dc current) is plotted versus the NBI fraction, which gives the net momentum input to the plasma, $f_{\mathrm{NBI}}=\left(P_{\mathrm{co}}-P_{\mathrm{cntr}}\right) /\left(P_{\mathrm{co}}+P_{\mathrm{cntr}}\right)$. The right figure shows that the angular velocity depends linearly on the net momentum input given by the NBI fraction. The data shown has been measured for an electron density of $1.5 \times 10^{19} \mathrm{~m}^{-3}$. The threshold for all scans exhibits a minimum which is located at a considerable amount of net co-injection, i.e. when the plasma fluid rotates in the direction of the plasma current. The scans at higher density require a higher value of the external perturbation field for mode onset, in accordance with the dependence of the error field threshold on electron density.

The minima in the mode threshold occur when the MHD frequency of the $2 / 1$ mode is equal to the frequency of the external perturbation field, $f_{\mathrm{DED}}=f_{\mathrm{MHD}}=f_{\Phi}-f^{*}$, where 


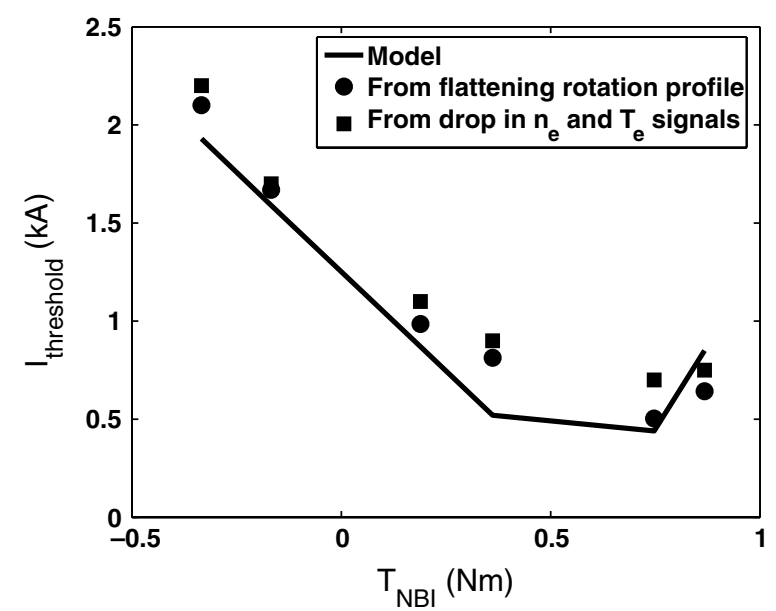

Figure 3. Threshold of field penetration estimated from a simple force balance model (line). The experimental data for plasma braking (circles) and mode formation (squares) are shown.

$f_{\Phi}$ is the toroidal rotation frequency and $f_{\mathrm{e}}^{*}$ is the electron diamagnetic drift frequency. This is directly verified using the dynamic option of the DED. The filled symbols in figure 2 are measured using an ac perturbation field with a frequency of $1 \mathrm{kHz}$. The direction of rotation of the field was opposite to the plasma current, i.e. in the electron diamagnetic drift direction. No mode excitation, even at the maximum possible coil current, has been obtained when the ac field rotated in the co-current direction.

The minimum in the mode excitation threshold for the data taken at an electron density of $1.5 \times 10^{19} \mathrm{~m}^{-3}$ are at a toroidal rotation frequency (measured by charge exchange recombination spectroscopy) of $1.7 \mathrm{kHz}$ for the dc case and at $0.7 \mathrm{kHz}$ for the ac case. The ac curve is shifted to the electron drift direction by the same amount as the applied rotation frequency of the external field, showing that the frequency match condition holds and the field penetration threshold is lowest when the MHD frequency of the mode is zero in the frame of the perturbation field and no or little braking of the plasma rotation is required for mode penetration [14].

This experimental finding has been modelled using a linear MHD model taking into account a single mode $(m / n=2 / 1)$ only. The results are in good agreement with the experiment [15]. The calculated force transfer to the plasma is maximum when the resonance condition is fulfilled. The same result can be obtained within a simple model balancing the forces acting on the plasma: (i) the momentum input by the heating beams in the centre, (ii) the friction due to charge exchange with neutrals at the edge, (iii) the force arising from edge ergodization and (vi) the ponderomotive force which tries to synchronize the MHD frequency of the mode with the frequency of the external perturbation field. Force (iii) acts always in the co-current direction [16], (ii) is a friction force which always decelerates. The ergodization force (iii) arises from the $E_{r} \times B_{\Theta}$ drift resulting from an outward directed electric field which is generated due to the enhanced electron losses in the ergodized region. Theory predicts a dependence of this force on the current in the ergodization coils, which saturates for high current: $F_{\text {erg }}=C I_{\mathrm{DED}}^{8 / 3} /\left(D+I_{\mathrm{DED}}^{8 / 3}\right)$ [17]. The parameters $C$ and $D$ depend on the temperature and density gradients in the ergodic region and can be determined from rotation measurements [18]. The sign of (i) is determined by the net momentum from co- and counter-injection and the sign of (vi) depends on whether the plasma needs to be accelerated or decelerated in order to match frequencies. The result of this calculation is shown in figure 3 . 
The critical DED current for mode generation is plotted as a function of the net momentum transfer from the NBI systems. The circles give the experimental values when plasma braking has been observed and the squares denote the critical field at the time of mode formation. The line shows the results of the model calculation. The experimentally found behaviour that the threshold has a minimum at medium values of co-momentum transfer is well reproduced by this simple force balance model.

In many of the experiments the locked $2 / 1$ mode is found not to spin up immediately after the DED coil current is reduced to zero but to stay locked for a longer time. The delay depends on the applied heating scenario and can last until the neutral beam power is finally switched off. The perturbation field current when unlocking of the mode occurs (measured in a power scan using ICRH) shows a linear dependence on the poloidal beta. The frequency of the $2 / 1$ mode after spin-up exhibits the same behaviour. It is very likely that this effect is not caused by a stabilizing influence of beta, but is due to the increase in the diamagnetic drift (and hence the decrease in the ponderomotive force [18]) due to the pressure rise, which shifts the diamagnetic frequency further in the counter current direction.

Furthermore, the dynamic operation of the DED has been applied to unlock a locked mode which was deliberately excited using a very low electron density during plasma startup. The island spins up to the frequency of the DED field when the perturbation field strength exceeds a certain value and the island width is found to be reduced from about $8 \mathrm{~cm}$ when it is locked down to $6 \mathrm{~cm}$ for the rotating island [19].

\section{ECRH / ECCD mode stabilization}

TEXTOR is equipped with a $140 \mathrm{GHz}, 800 \mathrm{~kW}$ gyrotron with a steerable launcher which allows the investigation of mode stabilization by ECRH and ECCD. For these studies the reproducible mode excitation as shown in figure 1 using a $1 \mathrm{kHz}$ ac DED current rotating in the counter-current direction has been applied.

The radial location of the island O-point in the plasma has been determined by various diagnostics and the phase of the tearing mode has been derived directly from the external DED currents. The deposition radius of the ECRH has been chosen by setting the elevation angle of the steerable launcher and depositing the radiation at a constant magnetic field along a vertical line. The amount of current drive has been adjusted by choosing an appropriate azimuthal launcher angle. The mode width has been determined either from an SXR camera or from ECE and magnetic measurements. The degree of mode suppression has been determined by normalization of the mode width to the value before ECRH/ECCD application. Various systematic scans of the mode suppression ratio have been performed in order to compare (i) ECRH with ECCD, (ii) $\mathrm{cw}$ heating with pulsed heating at various duty cycles and (iii) the difference between heating in the X-and O-points of the island [20]. The strongest suppression is found when pulsed ECRH is applied within the O-point of the island.

Figure 4 shows an example, where the ratio of mode suppression has been measured as a function of the toroidal injection angle which acts as a qualitative measure of the driven current. Negative angles indicate co-current drive, at positive angles the driven current is in counter direction to the plasma current. The gyrotron output power has been reduced to $200 \mathrm{~kW}$ in order to enhance the influence of plasma heating by the ECRH beam. The figure shows only slight differences between pure heating (around $0^{\circ}$ degrees) and current drive. For TEXTOR the main mechanism of mode suppression is due to heating in the island, which reduces the resistivity and results in more current flowing at the O-point. With the recently installed ECE 


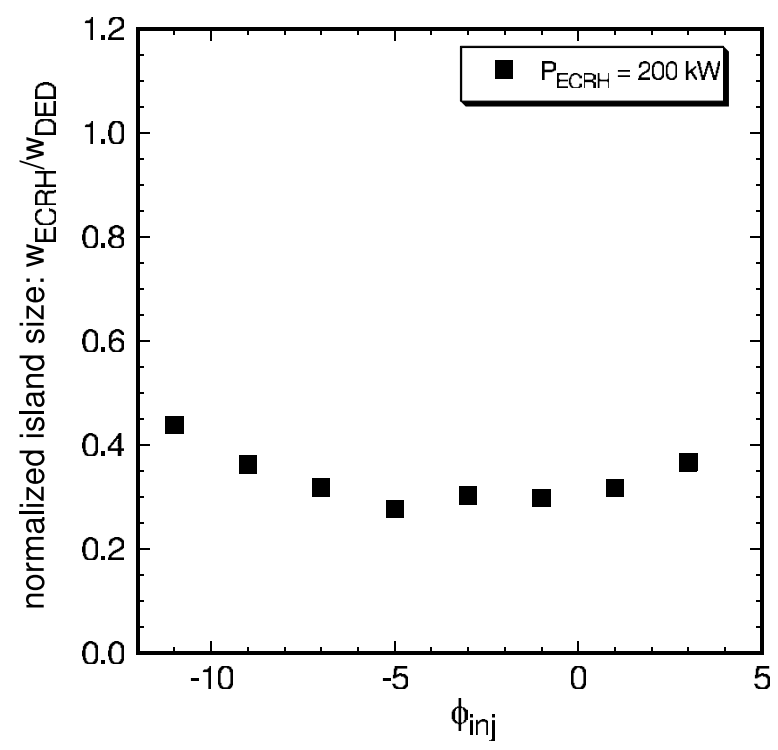

Figure 4. Ratio of island widths (measure of mode suppression) versus the toroidal injection angle (measure of the driven current) of the ECRH beam. The scan has been performed at low power in order to enhance the influence of plasma heating.

imaging diagnostic [21] a temperature peaking within the O-point of the island has indeed been proven.

From these results one can conclude that the NTM stabilization by ECCD on ITER will benefit from an additional influence of direct heating in the island, especially in the early stage of the mode suppression when the island size is still large. Depending on the peakedness of the temperature profile within the island, which is determined by the heat transport coefficient, the current density increase can be of the same order as the driven current (for $\chi_{\mathrm{e}} \approx 0.1 \mathrm{~m}^{2} \mathrm{~s}^{-1}$ ), or be at least a significant part of it when $\chi_{\mathrm{e}}$ is larger.

\section{Alfvén-like modes excited by field line perturbations}

Figure 5 shows on top a spectrogram of a magnetic pick-up coil. The time when the DED is operated at $1 \mathrm{kHz}$ ac current is indicated in the figure. At $t=3 \mathrm{~s}$ the DED is switched off and the $m / n=2 / 1$ tearing mode which was created phase locked to the DED field spins up. There are clearly visible bands of MHD modes in the frequency range between 13 and $27 \mathrm{kHz}$. The mode numbers determined by phase comparison of adjacent toroidally or poloidally separated magnetic pick-up coils are $n=1$ and $m=2$. Similar modes were recently found on FTU to occur when large 2/1 islands were present in the plasma [22]. A scaling between TEXTOR and FTU revealed that the frequency of these modes scales with the magnetic field and the ion density like an Alfvén wave, $f \propto B / \sqrt{m_{\mathrm{i}} n_{\mathrm{i}}}$ [23]. A recent isotope scan on TEXTOR, where H, D and He plasmas were compared validated this scaling with respect to the isotope mass [24]. Figure 5 shows that the bands appear before the island is formed (left arrow, compare bottom trace which gives the island size determined by the SXR). This new finding questions the conclusion given in [22] that the Alfvén waves travel within the island. Obviously, a flux surface perturbation above a certain level of external resonant perturbation field is able to create these modes. 


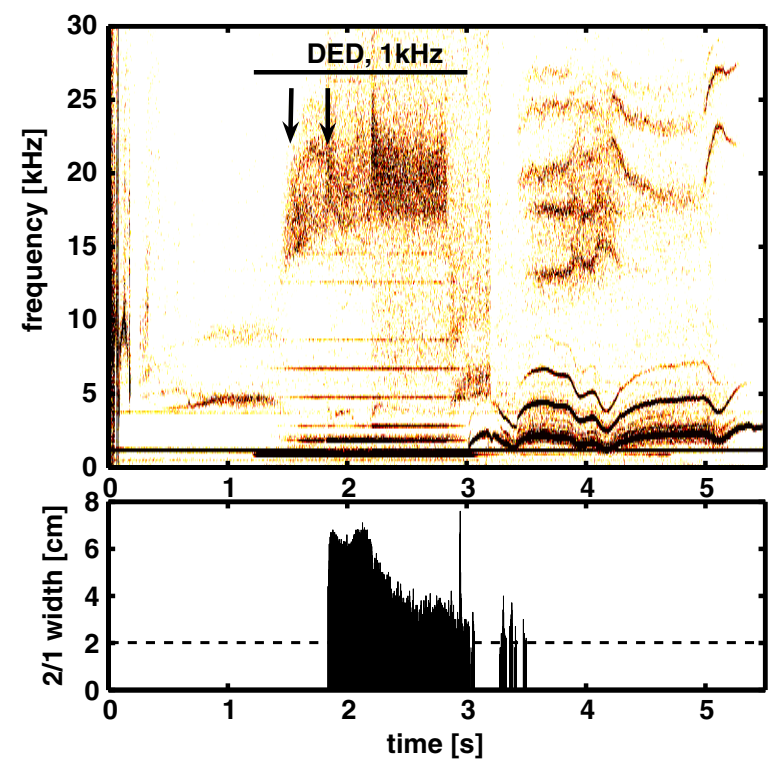

Figure 5. Magnetic spectrogram (top) and width of the $m / n=2 / 1$ island (bottom). The onset of the Alfvén-like modes occurs well before the island is formed.

(This figure is in colour only in the electronic version)

\section{Summary and conclusions}

The TEXTOR tokamak is equipped with a DED which allows the reproducible excitation of $m / n=2 / 1$ modes which are created either locked in the tokamak frame in dc operation or rotating with the external perturbation field when ac operation is applied. In combination with the flexible heating systems (co- and counter-NBI with continuously adjustable power, ECRH with steerable launcher) detailed studies of mode generation by the external field and suppression by ECRH and ECCD can be done.

The dependence of the mode threshold on the plasma fluid rotation velocity shows a minimum when the plasma rotates in the co-current direction. This is explained by the resonance condition that the MHD frequency of the mode has to be equal to the frequency of the external field. The dynamic operation of the DED allowed a direct proof of this.

The mode suppression on TEXTOR is mainly due to heating in the island. ITER will have a strong contribution from this effect, especially at large island sizes in the first stage of mode suppression.

A new class of Alfvén-like modes has been identified which are created by flux surface perturbations, either due to the external error field or due to a large $2 / 1$ island in the plasma.

\section{References}

[1] Rutherford P H 1973 Phys. Fluids 161903

[2] ITER Physics Expert Group on Disruptions, Plasma Control and MHD and ITER Physics Base Editors, ITER EDA Nucl. Fusion 392251

[3] Sauter O et al 1997 Phys. Plasmas 41654

[4] Hender T C et al 1992 Nucl. Fusion 322091

[5] La Haye R J et al 1992 Nucl. Fusion 322119 
[6] Buttery R J et al 1999 Nucl. Fusion 391827

[7] Buttery R J et al 2000 Nucl. Fusion 40807

[8] Wolfe S M et al 2005 Phys. Plasmas 12056110

[9] Finken K H (ed) 1997 (special issue) Dynamic ergodic divertor Fusion Eng. Des. 37335

[10] Koslowski H R et al 2004 Proc. 31st EPS Conf. Plasma Physics (London, UK) vol 28G ECA P1.124

[11] Uhlemann R and Ongena J 1999 Fusion Technol. 3542

[12] Westerhof E et al 2003 Nucl. Fusion 431371

[13] Fitzpatrick R 1993 Nucl. Fusion 331049

[14] Koslowski H R et al 2006 Nucl. Fusion 46 L1

[15] Kikuchi Y et al 2006 Plasma Phys. Control. Fusion 48169

[16] Finken K H et al 2005 Phys. Rev. Lett. 94015003

[17] Kaganovich I and Rozhansky V 1998 Phys. Plasmas 53901 Abdullaev S S et al 1999 Phys. Plasmas 6153

[18] Bock M de 2007 Understanding and controlling plasma rotation in Tokamaks PhD Thesis TU Eindhoven

[19] Liang Y et al 2004 Proc. 31st EPS Conf. Plasma Physics (London, UK) 28G ECA P1.126

[20] Westerhof E et al 2006 Nucl. Fusion 46 at press

[21] Wang J et al 2004 Rev. Sci. Instrum. 753875

[22] Buratti P et al 2005 Nucl. Fusion 451446

[23] Buratti P et al 2005 Proc. 32nd EPS Conf. Plasma Physics (Tarragona, Spain) P5.055

[24] Zimmermann O et al 2006 Proc. 33rd EPS Conf. Plasma Physics (Rome, Italy) P2.156 